\title{
Fractional Ginzburg-Landau equation for fractal media
}

\author{
Vasily E. Tarasov ${ }^{\mathrm{a}, *}$, George M. Zaslavsky ${ }^{\mathrm{b}, \mathrm{c}}$ \\ ${ }^{a}$ Skobeltsyn Institute of Nuclear Physics, Moscow State University, Moscow 119992, Russian Federation \\ ${ }^{\mathrm{b}}$ Courant Institute of Mathematical Sciences, New York University, 251 Mercer St., New York, \\ NY 10012, USA \\ ${ }^{\mathrm{c}}$ Department of Physics, New York University, 2-4 Washington Place, New York, NY 10003, USA
}

Available online 8 April 2005

\begin{abstract}
We derive the fractional generalization of the Ginzburg-Landau equation from the variational Euler-Lagrange equation for fractal media. To describe fractal media we use the fractional integrals considered as approximations of integrals on fractals. Some simple solutions of the Ginzburg-Landau equation for fractal media are considered and different forms of the fractional Ginzburg-Landau equation or nonlinear Schrödinger equation with fractional derivatives are presented. The Agrawal variational principle and its generalization have been applied.
\end{abstract}

(C) 2005 Elsevier B.V. All rights reserved.

PACS: 03.40.-t; 05.45.Df; 47.53. + n

Keywords: Fractional equation; Fractional derivatives and integrals fractal medium; Ginzburg-Landau equation

\section{Introduction}

We call the fractional equation an equation that contains fractional derivatives or integrals. Derivatives and integrals of fractional order have found many applications

\footnotetext{
*Corresponding author. Tel.: + 7095 9395989; fax: + 70959390397.

E-mail address: tarasov@theory.sinp.msu.ru (V.E. Tarasov).
} 
in recent studies in physics. The interest in fractional equations has been growing continually during the last few years because of numerous applications: kinetic theories of systems with chaotic dynamics [1,2]; pseudochaotic dynamics [3]; dynamics in a complex or porous media [4]; random walks with a memory and flights [5-7]; and many others.

Fractional integrals and derivatives can be used to describe processes with different levels of complexity. The new type of problems has rapidly increased interest in areas in which the fractal features of a process or the medium impose a necessity of applying tools that are non-traditional in "regular" smooth physical equations. In many problems the real fractal structure of matter can be disregarded and the medium can be replaced by some smoothed continuous mathematical model where fractional integrals appear [8]. The order of the fractional integral is equal to the fractal mass dimension of the medium and in this way one can take into account the fractality of the media. It was proved that integrals on a net of fractals can be approximated by fractional integrals [9] and that fractional integrals can be considered as integrals over the space with fractional dimension up to a numerical factor [10]. The last proof used the formulas of dimensional regularizations [11].

The fractional generalization of the Ginzburg-Landau equation was suggested in Ref. [12]. This equation can be used to describe the dynamical processes in a medium with fractal dispersion. Since the fractals can be realized in nature as a fractal process or fractal media, it is interesting to derive the fractional Ginzburg-Landau (FGL) equation using a corresponding generalization of the free energy functional.

It is known [13] that the Ginzburg-Landau equation

$$
g \Delta Z-a Z-b Z^{3}=0
$$

can be derived as the variational Euler-Lagrange equation

$$
\frac{\delta F\{Z(\mathbf{x})\}}{\delta Z(\mathbf{x})}=0
$$

for the free energy functional

$$
F\{Z(\mathbf{x})\}=F_{0}+\frac{1}{2} \int_{W}\left[g(\nabla Z)^{2}+a Z^{2}+\frac{b}{2} Z^{4}\right] \mathrm{d} V_{3},
$$

where the integration is over the three-dimensional region $W$ of continuous media. Here $F_{0}$ is a free energy of the normal state, i.e., $F\{Z\}$ for $Z=0$. In this paper we consider a fractional generalization of (2) that can appear from two places: fractional generalization of the integral in Eq. (2) and fractional generalization of the derivatives in Eq. (2). Different forms of the FGL equation are considered in the coordinate space and in the dual space after applying the Fourier transform. Finally, the Agrawal variational principle [14] and its generalization have been applied to obtain the FGL equation. 


\section{Fractional generalization of free energy functional}

Let us consider the thermodynamic potential (free energy functional) $F\{Z(\mathbf{x})\}$ for the non-equilibrium state of the fractal medium, where $Z(\mathbf{x})$ is a scalar field. The free energy functional has the form

$$
F\{Z(\mathbf{x})\}=F_{0}+\int_{W} \mathscr{F}(Z(\mathbf{x}), \nabla Z(\mathbf{x})) \mathrm{d} V_{3},
$$

where $\mathscr{F}(Z(\mathbf{x}), \nabla Z(\mathbf{x}))$ is the free energy density; $\nabla Z=\partial Z / \partial \mathbf{x}$. For the GinzburgLandau potential (2) this density is

$$
\mathscr{F}(Z(\mathbf{x}), \nabla Z(\mathbf{x}))=\frac{1}{2}\left[g(\nabla Z)^{2}+a Z^{2}+\frac{b}{2} Z^{4}\right] .
$$

The simplest fractional generalization of (2) can be written in the form

$$
F\{Z(\mathbf{x})\}=F_{0}+\int_{W} \mathscr{F}(Z(\mathbf{x}), \nabla Z(\mathbf{x})) \mathrm{d} V_{D} .
$$

Here $D$ is a fractal mass dimension of the fractal medium, and $\mathrm{d} V_{D}$ is an element of the $D$-dimensional volume:

$$
\mathrm{d} V_{D}=C_{3}(D, \mathbf{x}) \mathrm{d} V_{3} .
$$

Note that the interpretation of the fractional integration is connected with fractional dimension [10], which follows from the well-known formulas for dimensional regularizations [11]: the fractional integral can be considered as an integral in the fractional dimension space up to the numerical factor $\Gamma(D / 2) /\left(2 \pi^{D / 2} \Gamma(D)\right)$.

For the Riesz definition of fractional integral, the function $C_{3}(D, \mathbf{x})$ is

$$
C_{3}(D, \mathbf{x})=\frac{2^{3-D} \Gamma(3 / 2)}{\Gamma(D / 2)}|\mathbf{x}|^{D-3} .
$$

The initial point of the fractional integral is set to zero and the numerical factor in Eq. (6) gives the usual integral in the limit $D \rightarrow(3-0)$. Note that the numerical factor $\gamma_{3}^{-1}(D)=\Gamma(1 / 2) / 2^{D} \pi^{3 / 2} \Gamma(D / 2)$ used in Ref. [15] leads to $\gamma_{3}^{-1}(3-0)=$ $\Gamma(1 / 2) / 2^{3} \pi^{3 / 2} \Gamma(3 / 2)$ in the limit $D \rightarrow(3-0)$.

For the Riemann-Liouville fractional integral, the function $C_{3}(D, \mathbf{x})$ is

$$
C_{3}(D, \mathbf{x})=\frac{\left|x_{1} x_{2} x_{3}\right|^{D / 3-1}}{\Gamma^{3}(D / 3)} .
$$

In Cartesian's coordinates $x_{k}$,

$$
\mathbf{x}=\sum_{k=1}^{3} x_{k} \mathbf{e}_{k}, \quad \mathrm{~d} V_{3}=\mathrm{d} x_{1} \mathrm{~d} x_{2} \mathrm{~d} x_{3} .
$$

The simplest fractional generalization of (2) for fractal media is

$$
F\{Z(\mathbf{x})\}=F_{0}+\frac{1}{2} \int_{W}\left[g(\nabla Z)^{2}+a Z^{2}+\frac{b}{2} Z^{4}\right] \mathrm{d} V_{D} .
$$


Eq. (8) can be considered as terms of the expansion of a functional in series over small values $Z(\mathbf{x})$ and the integer derivatives $\nabla Z=\partial Z / \partial \mathbf{x}$.

For homogeneous media without external fields the parameter $Z$ does not depend on coordinates and Eq. (8) gives

$$
F\{Z\}=F_{0}+\frac{a V_{D}}{2} Z^{2}+\frac{b V_{D}}{4} Z^{4},
$$

where $V_{D}$ is a $D$-dimensional volume of fractal medium in the region $W$. The equilibrium value of $Z$ corresponds to the minimum condition for (9):

$$
\mathrm{d} F\{Z\} / \mathrm{d} Z=0 .
$$

If $a / b>0$, then the free energy potential has the single minimum $Z=0$. If $a / b<0$, then there are two minima $Z= \pm \sqrt{-a / b}$. The phase transition is realized when $a / b$ passes through zero.

\section{Ginzburg-Landau equation for fractal media}

In the general case, the equilibrium value of $Z(\mathbf{x})$ is defined by the condition of the minimum of $F\{Z(\mathbf{x})\}$, which has the form of variational Euler-Lagrange (1). Using functional (4) with the free energy density $\mathscr{F}(Z(\mathbf{x}), \nabla Z(\mathbf{x}))$, we get the EulerLagrange equation

$$
C_{3}(D, \mathbf{x}) \frac{\partial \mathscr{F}}{\partial Z}-\sum_{k=1}^{3} \nabla_{k}\left(C_{3}(D, \mathbf{x}) \frac{\partial \mathscr{F}}{\partial \nabla_{k} Z}\right)=0
$$

where $C_{3}(D, \mathbf{x})$ is defined by Eq. (6) or (7), and $\nabla_{k}=\partial / \partial x_{k}, k=1,2,3$. Using (8), we get the equation

$$
g C_{3}^{-1}(D, \mathbf{x}) \nabla_{k}\left(C_{3}(D, \mathbf{x}) \nabla_{k} Z\right)-a Z-b Z^{3}=0
$$

that can be considered as the Ginzburg-Landau equation for fractal media in the case $\mathscr{F}=\mathscr{F}(Z(\mathbf{x}), \nabla Z(\mathbf{x}))$.

Eq. (11) can be rewritten in an equivalent form

$$
g \Delta Z+E_{k}(D, \mathbf{x}) \nabla_{k} Z-a Z-b Z^{3}=0,
$$

where

$$
E_{k}(D, \mathbf{x})=C_{3}^{-1}(D, \mathbf{x}) \nabla_{k} C_{3}(D, \mathbf{x}) .
$$

In the one-dimensional case, when $0<D \leqslant 1$, we have

$$
Z_{x x}^{\prime \prime}+\frac{D-1}{g|x|} Z_{x}^{\prime}-(a / g) Z-(b / g) Z^{3}=0,
$$

where $x=x_{1}$ and $Z_{x}^{\prime}=\mathrm{d} Z / \mathrm{d} x_{1}$. The simplest solutions of this equation are considered in Section 7. Note that this equation has an analog in the form of an equation for a nonlinear oscillator with friction. Therefore, the Ginzburg-Landau 
equation for fractal media describes dissipative nonlinear oscillations in coordinate space.

In the general case, the free energy density can also depend on fractional derivatives of $Z$. In this case we can use the fractional generalization of the Agrawal variational equation [14]. This case will be considered in Section 6 .

\section{Ginzburg-Landau equation with fractional derivatives}

Let us recall the appearance of the Ginzburg-Landau equation with fractional derivatives suggested in Ref. [12]. Consider wave propagation in some media and present the wave vector $\mathbf{k}$ in the form

$$
\mathbf{k}=\mathbf{k}_{0}+\mathbf{\kappa}=\mathbf{k}_{0}+\mathbf{\kappa}_{\|}+\mathbf{\kappa}_{\perp},
$$

where $\mathbf{k}_{0}$ is the unperturbed wave vector and subscripts $(\|, \perp)$ are taken respectively to the direction of $\mathbf{k}_{0}$. Considering a symmetric dispersion law $\omega=\omega(k)$ for wave propagation with $\kappa \ll k_{0}$, we have

$$
\omega(k)=\omega\left(\left|\mathbf{k}_{0}+\mathbf{\kappa}\right|\right) \approx \omega\left(k_{0}\right)+c\left(\left|\mathbf{k}_{0}+\mathbf{\kappa}\right|-k_{0}\right) \approx \omega\left(k_{0}\right)+c \kappa_{\|}+\frac{c}{2 k_{0}} \kappa_{\perp}^{2},
$$

where $c=\partial \omega / \partial k_{0}$. Expression (15) in dual space ("momentum representation") corresponds to the following equation in coordinate space,

$$
-\mathrm{i} \frac{\partial Z}{\partial t}=\mathrm{i} c \frac{\partial Z}{\partial x_{1}}+\frac{c}{2 k_{0}} \Delta Z
$$

with respect to the field $Z=Z(\mathbf{x}, t)$, where $x_{1}$ is along $\mathbf{k}_{0}$. Here we use the operator correspondence between the dual space and the usual space-time:

$$
v \equiv \omega(k) \longleftrightarrow \mathrm{i} \frac{\partial}{\partial t}, \quad \kappa_{\|} \longleftrightarrow-\mathrm{i} \frac{\partial}{\partial x_{1}}, \quad\left(\boldsymbol{\kappa}_{\perp}\right)^{2} \longleftrightarrow-\Delta=-\frac{\partial^{2}}{\partial x_{2}}-\frac{\partial^{2}}{\partial x_{3}} .
$$

A generalization to the nonlinear case can be carried out as in (15) through a nonlinear dispersion law dependence on the wave amplitude:

$$
\omega=\omega\left(k,|Z|^{2}\right) \approx \omega(k, 0)+b|Z|^{2}=\omega\left(\left|\mathbf{k}_{0}+\mathbf{\kappa}\right|, 0\right)+b|Z|^{2}
$$

with some constant $b=\partial \omega\left(k,|Z|^{2}\right) / \partial|Z|^{2}$ at $|Z|^{2}=0$. In analogy with (16), the nonlinear equation takes the form

$$
-\mathrm{i} \frac{\partial Z}{\partial t}=\mathrm{i} c \frac{\partial Z}{\partial x}+\frac{c}{2 k_{0}} \Delta Z-\omega\left(k_{0}\right) Z-b|Z|^{2} Z .
$$

This equation is also known as the nonlinear Schrödinger (NLS) equation in which any of the coefficients may be complex. Indeed, for a traveling wave $Z=Z\left(x_{1}-\right.$ $\left.c t, x_{2}, x_{3}\right)$ we have

$$
-\mathrm{i} c \frac{\partial Z}{\partial \xi}=\frac{c}{2 k_{0}} \Delta Z-\omega_{0} Z-b|Z|^{2} Z
$$

with $\xi=x_{1}-c t, \omega_{0}=\omega\left(k_{0}\right)$. 
Wave propagation in media with fractal properties can be easily generalized by rewriting the dispersion law (15). Namely, one can replace (15) and (18) by the following equation in dual space:

$$
\omega\left(k,|Z|^{2}\right)=\omega\left(k_{0}, 0\right)+c \kappa_{\|}+c_{\alpha}\left(\boldsymbol{\kappa}_{\perp}^{2}\right)^{\alpha / 2}+b|Z|^{2}
$$

with a fractional value of $1<\alpha<2$ and a new constant $c_{\alpha}$.

Using the connection between fractional derivative and its Fourier transform [15]

$$
(-\Delta)^{\alpha / 2} \longleftrightarrow\left(\boldsymbol{\kappa}_{\perp}^{2}\right)^{\alpha / 2}
$$

we obtain the equation

$$
-\mathrm{i} \frac{\partial Z}{\partial t}=\mathrm{i} c \frac{\partial Z}{\partial x}-\frac{c}{2 k_{0}}(-\Delta)^{\alpha / 2} Z-\omega_{0} Z-b|Z|^{2} Z,
$$

which corresponds to (21) in time-coordinate space. For traveling waves as in (20), we get the equation

$$
\text { ic } \frac{\partial Z}{\partial \xi}=\frac{c}{2 k_{0}}(-\Delta)^{\alpha / 2} Z+\omega_{0} Z+b|Z|^{2} Z
$$

that can be called nonstationary FGL equation or fractional nonlinear Schrödinger equation (FNLS). Let us comment on the physical structure of (24). The first term on the right-hand side is related to the wave propagation in a medium with fractal properties. The fractional derivative may also appear as a result of ray chaos $[16,17]$ or due to superdiffusive wave propagation (see also the discussion in Refs. $[1,16]$ and corresponding references therein). Other terms on the right-hand side of Eqs. (23) and (24) correspond to wave interaction due to the nonlinear properties of the media. Thus, Eq. (24) can describe fractal processes of self-focusing and related issues.

We may consider a one-dimensional simplification of (24), i.e.,

$$
c \frac{\partial Z}{\partial \xi}=g \mathbf{D}_{x}^{\alpha} Z+a Z+b|Z|^{2} Z
$$

with some generalized constants $g, b, c$, and the Riesz fractional derivative

$$
\left(\mathbf{D}_{y}^{\alpha} f\right)(y)=\frac{-1}{2 \cos (\pi \alpha / 2)}\left(\frac{1}{\Gamma(1-\alpha)} \frac{\mathrm{d}}{\mathrm{d} y} \int_{-\infty}^{y} \frac{f(z) \mathrm{d} z}{(y-z)^{\alpha}}+\frac{-1}{\Gamma(1-\alpha)} \frac{\mathrm{d}}{\mathrm{d} y} \int_{y}^{+\infty} \frac{f(z) \mathrm{d} z}{(z-y)^{\alpha}}\right) .
$$

As a particular case, we can reduce Eq. (25) to a stationary solution $Z=Z(x)$. In this case, Eq. (25) takes the form of the stationary FGL:

$$
g \mathbf{D}_{x}^{\alpha} Z+c \mathbf{D}_{x}^{1} Z+a Z+b|Z|^{2} Z=0
$$

or for real $Z$ :

$$
g \mathbf{D}_{x}^{\alpha} Z+c \mathbf{D}_{x}^{1} Z+a Z+b Z^{3}=0 .
$$


Eq. (28) differs from the fractional Burgers equation [18,19] in the structure of the nonlinear term. Nevertheless, an analysis similar to that in Refs. [18,19] may be performed to obtain some estimates on the solution.

It is well known that the nonlinear term in equations of the type (20) leads to a steepening of the solution and its singularity. The steepening process may be stopped by a diffusional or dispersional term, i.e. by a higher derivative term. A similar phenomenon may appear for the fractional nonlinear equations (25), (27). It has been shown in Ref. [19] that for the fractional Burgers equation there exists a critical value $\alpha_{c}$ such that the solution is regular for all $x$ if $\alpha>\alpha_{c}$.

\section{Ginzburg-Landau equations with fractional derivatives in dual space}

In order to derive the FGL equations from a variational principle, we can use two methods. One of them was suggested in Ref. [10] and will be considered here. The second one is a generalization of the Agrawal variational [14] for the fractal media, and will be considered in Section 6.

Let us introduce the derivatives with a fractional power of coordinates $x_{k}^{\beta}$ :

$$
\frac{\partial}{\partial x_{k}^{\beta}}=\frac{\left|x_{k}\right|^{1-\beta}}{\beta} \frac{\partial}{\partial x_{k}} \text {. }
$$

These derivatives transform into fractional derivatives in dual space ("momentum representation"). The Fourier transform of the coordinates $\left|x_{k}\right|^{1-\beta}$ leads us to the Reisz fractional derivative

$$
\left|x_{k}\right|^{1-\beta} \longleftrightarrow-\mathbf{D}_{y_{k}}^{1-\beta}
$$

where we use definition (26). The Fourier transform of Eq. (29) is

$$
\frac{\partial}{\partial x_{k}^{\beta}}=\frac{\left|x_{k}\right|^{1-\beta}}{\beta} \frac{\partial}{\partial x_{k}} \longleftrightarrow-\frac{1}{\beta} \mathbf{D}_{y_{k}}^{1-\beta} y_{k}
$$

Consider free energy functional $\mathscr{F}=\mathscr{F}\left(Z, \partial Z / \partial x_{k}^{\beta}\right)$ for the one-dimensional case. Then the FGL equation for fractal media is of the form

$$
g C_{1}^{-1}(\beta, x) \frac{\partial}{\partial x^{\beta}}\left(C_{1}(\beta, x) \frac{\partial}{\partial x^{\beta}} Z\right)-a Z-b Z^{3}=0,
$$

where $C_{1}(\beta, x)=|x|^{\beta-1} / \Gamma(\beta)$, or in an equivalent form

$$
\frac{g}{\beta^{2}}|x|^{2-2 \beta} \frac{\partial^{2}}{\partial x^{2}} Z-a Z-b Z^{3}=0 .
$$

After Fourier transform of this equation, we obtain the FGL equation in dual space:

$$
\frac{g}{\beta^{2}} \mathbf{D}_{y}^{2-2 \beta}\left(y^{2} \tilde{Z}\right)+a \tilde{Z}+b \tilde{Z}^{3}=0 .
$$


Here $\tilde{Z}(y)$ is a Fourier transform of $Z(x)$, i.e., $Z(x) \longleftrightarrow \tilde{Z}(y)$. For the field $\Psi(y)=y^{2} \tilde{Z}(y)$, we can rewrite Eq. (33) in the form

$$
g \mathbf{D}_{x}^{\alpha} \Psi(x)+a(x) \Psi(x)+b(x) \Psi^{3}(x)=0,
$$

where $\alpha=2-2 \beta, 0<\alpha<2$ and $a(x)=a \beta^{2} /|x|^{2}, b(x)=b \beta^{2} /|x|^{6}$.

As a result, the FGL equation for fractal media has fractional derivatives in dual space. The FGL equation with fractional derivatives in coordinate space is considered in the next section.

\section{Ginzburg-Landau equations with fractional derivatives from the fractional Agrawal principle}

Let us, first, extend the fractional Agrawal variational principle [14] for the case of fractal media.

The simplest generalization of the free energy functional with fractional integrals and derivatives can be written in the form

$$
F\{Z(\mathbf{x})\}=F_{0}+\int_{W} \mathscr{F}\left(Z(\mathbf{x}), \mathbf{D}^{\alpha} Z(\mathbf{x})\right) \mathrm{d} V_{D} .
$$

Here $D$ is a fractal mass dimension of fractal medium, and $\mathrm{d} V_{D}$ is an element of $D$ dimensional volume such that $\mathrm{d} V_{D}=C_{3}(D, \mathbf{x}) \mathrm{d} V_{3}$. The function $C_{3}(D, \mathbf{x})$ is defined by relations (6) and (7). $\mathbf{D}^{\alpha}$ is a fractional derivative that is defined by Eq. (26). The potential density is

$$
\mathscr{F}=\frac{1}{2}\left[g\left(\mathbf{D}^{\alpha} Z\right)^{2}+a Z^{2}+\frac{b}{2} Z^{4}\right] .
$$

The condition of the minimum of free energy potential (34), (35) gives the Euler-Lagrange equation in the form

$$
C_{3}(D, \mathbf{x}) \frac{\partial \mathscr{F}}{\partial Z}+\sum_{k=1}^{3} \mathbf{D}_{x_{k}}^{\alpha}\left(C_{3}(D, \mathbf{x}) \frac{\partial \mathscr{F}}{\partial \mathbf{D}_{x_{k}}^{\alpha} Z}\right)=0 .
$$

In general, $D \neq 3 \alpha$ and Eq. (36) gives

$$
g C_{3}^{-1}(D, \mathbf{x}) \sum_{k=1}^{3} \mathbf{D}_{x_{k}}^{\alpha}\left(C_{3}(D, \mathbf{x}) \mathbf{D}_{x_{k}}^{\alpha} Z\right)+a Z+b Z^{3}=0 .
$$

It can be considered as a fractional generalization of the Ginzburg-Landau equation for fractal media. Consider some cases of this equation.

1. In the one-dimensional case $Z=Z(x)$, the coordinate fractional derivative is $\mathbf{D}_{x}^{\alpha}$, i.e.,

$$
\mathscr{F}=\mathscr{F}\left(Z, \mathbf{D}_{x}^{\alpha} Z\right) .
$$


The FGL potential density has the form

$$
\mathscr{F}=\frac{1}{2} g\left(\mathbf{D}_{x}^{\alpha} Z\right)^{2}+\frac{a}{2} Z^{2}+\frac{b}{4} Z^{4} .
$$

Using the formulas for fractional integration by parts

$$
\begin{aligned}
& \int_{-\infty}^{\infty} f(x) \frac{\mathrm{d}^{\beta} g(x)}{\mathrm{d} x^{\beta}} \mathrm{d} x=\int_{-\infty}^{\infty} g(x) \frac{\mathrm{d}^{\beta} f(x)}{\mathrm{d}(-x)^{\beta}} \mathrm{d} x, \\
& \int_{-\infty}^{\infty} f(x) \mathbf{D}_{x}^{\alpha} g(x) \mathrm{d} x=\int_{-\infty}^{\infty} g(x) \mathbf{D}_{x}^{\alpha} f(x) \mathrm{d} x,
\end{aligned}
$$

we obtain the Euler-Lagrange equation

$$
\mathbf{D}_{x}^{\alpha}\left(C_{1}(D, x) \frac{\partial \mathscr{F}}{\partial \mathbf{D}_{x}^{\alpha}}\right)+C_{1}(D, x) \frac{\partial \mathscr{F}}{\partial Z}=0,
$$

where the function $C_{1}(D, x)$ is defined as

$$
C_{1}(D, x)=\frac{|x|^{D-1}}{\Gamma(D)} .
$$

Using for $\mathscr{F}$ (38), we arrive at

$$
C_{1}^{-1}(D, x) \mathbf{D}_{x}^{\alpha}\left(C_{1}(D, x) \mathbf{D}_{x}^{\alpha} Z\right)+a Z+b Z^{3}=0 .
$$

For the case $D=1$, we have $C_{1}=1$ and (41) transforms into

$$
\mathbf{D}_{x}^{2 \alpha} Z+a Z+b Z^{3}=0,
$$

where $\mathbf{D}_{x}^{\alpha}$ is the Riesz derivative.

2. The free energy functional density $\mathscr{F}$ can be a function of $Z=Z(x)$, and two different fractional derivatives $\mathbf{D}_{x}^{\alpha} Z$, and $\mathbf{D}_{x}^{\beta} Z, \alpha \neq \beta$, i.e.,

$$
\mathscr{F}=\mathscr{F}\left(Z, \mathbf{D}_{x}^{\alpha} Z, \mathbf{D}_{x}^{\beta} Z\right) .
$$

The FGL potential density has the form

$$
\mathscr{F}=\frac{1}{2} g_{1}\left(\mathbf{D}_{x}^{\alpha} Z\right)^{2}+\frac{1}{2} g_{2}\left(\mathbf{D}_{x}^{\beta} Z\right)^{2}+\frac{a}{2} Z^{2}+\frac{b}{4} Z^{4} .
$$

For example, $\alpha=1,0<\beta<1$. Using again (39), we obtain the following Euler-Lagrange equation,

$$
\mathbf{D}_{x}^{\alpha}\left(C_{1}(D, x) \frac{\partial \mathscr{F}}{\partial \mathbf{D}_{x}^{\alpha} Z}\right)+\mathbf{D}_{x}^{\beta}\left(C_{1}(D, x) \frac{\partial \mathscr{F}}{\partial \mathbf{D}_{x}^{\beta} Z}\right)+C_{1}(D, x) \frac{\partial \mathscr{F}}{\partial Z}=0,
$$

where $C_{1}(D, x)$ is given in (41). For the FGL density (44), we get the FGL equation for fractal media

$$
\begin{aligned}
& g_{1} C_{1}^{-1}(D, x) \mathbf{D}_{x}^{\alpha}\left(C_{1}(D, x) \mathbf{D}_{x}^{\alpha} Z\right)+g_{2} C_{1}^{-1}(D, x) \mathbf{D}_{x}^{\beta}\left(C_{1}(D, x) \mathbf{D}_{x}^{\beta} Z\right) \\
& \quad+a Z+b Z^{3}=0 .
\end{aligned}
$$


Particularly, when $D=1$ and $C_{1}=1$, we have

$$
g_{1} \mathbf{D}_{x}^{2 \alpha} Z+g_{2} \mathbf{D}_{x}^{2 \beta} Z+a Z+b Z^{3}=0, \quad 0 \leqslant \alpha, \beta \leqslant 1 .
$$

3. In the general case, the free energy density functional is a function of $Z=Z(\mathbf{x})$, and derivatives $\mathbf{D}_{x_{k}}^{\alpha_{k}} Z$ of fractional orders $\alpha_{k}$ different for coordinates $x_{k}$, i.e.,

$$
\mathscr{F}=\mathscr{F}\left(Z, \mathbf{D}_{x_{1}}^{\alpha_{1}} Z, \mathbf{D}_{x_{2}}^{\alpha_{2}} Z, \mathbf{D}_{x_{3}}^{\alpha_{3}} Z\right) .
$$

For this case, the FGL equation for fractal media is

$$
g C_{3}^{-1}(D, \mathbf{x}) \sum_{k=1}^{3} \mathbf{D}_{x_{k}}^{\alpha_{k}}\left(C_{3}(D, \mathbf{x}) \mathbf{D}_{x_{k}}^{\alpha_{k}} Z\right)+a Z+b Z^{3}=0 .
$$

The sum of orders $\alpha_{k}$ can be equal to the fractal mass dimension $D$ of the medium

$$
\alpha_{1}+\alpha_{2}+\alpha_{3}=D
$$

but in the general case it can be that $\alpha_{1}+\alpha_{2}+\alpha_{3} \neq D$.

\section{Simplest solutions}

Let us consider a simple example of the Ginzburg-Landau equation (11) for fractal media in the one-dimensional case. Then

$$
\frac{\partial Z}{\partial x_{2}}=0, \quad \frac{\partial Z}{\partial x_{3}}=0
$$

and $Z=Z\left(x_{1}\right)$. Eq. (11) for one-dimensional case and $0<D<1$ transforms into

$$
g C_{1}^{-1}(D, x) \frac{\partial}{\partial x}\left(C_{1}(D, x) \frac{\partial}{\partial x} Z\right)-a Z-b Z^{3}=0,
$$

where $x=x_{1}$, and $C_{1}(D, x)$ is defined by (41). This equation can be rewritten in an equivalent form

$$
Z_{x x}^{\prime \prime}+\frac{D-1}{g|x|} Z_{x}^{\prime}-(a / g) Z-(b / g) Z^{3}=0
$$

where $Z_{x}^{\prime}=\mathrm{d} Z(x) / \mathrm{d} x$. Note that Eq. (50) has an analog of the equation for a nonlinear oscillator with friction

$$
\ddot{x}(t)=\alpha x(t)+\beta x^{3}(t)-\gamma(t) \dot{x}(t),
$$

where we use

$$
\alpha=a / g, \quad \beta=b / g, \quad \gamma(t)=(D-1) / g|t| .
$$

Therefore the FGL equation for fractal media (49) describes coordinate nonlinear oscillations with a "dissipative"-like term.

Let us consider the solution of (49) with $b=0 . Z(\mathbf{x})$ satisfies the equation

$$
g\left[c_{1}(D, x) Z_{x}^{\prime}\right]_{x}^{\prime}-a C_{1}(D, x) Z=0,
$$




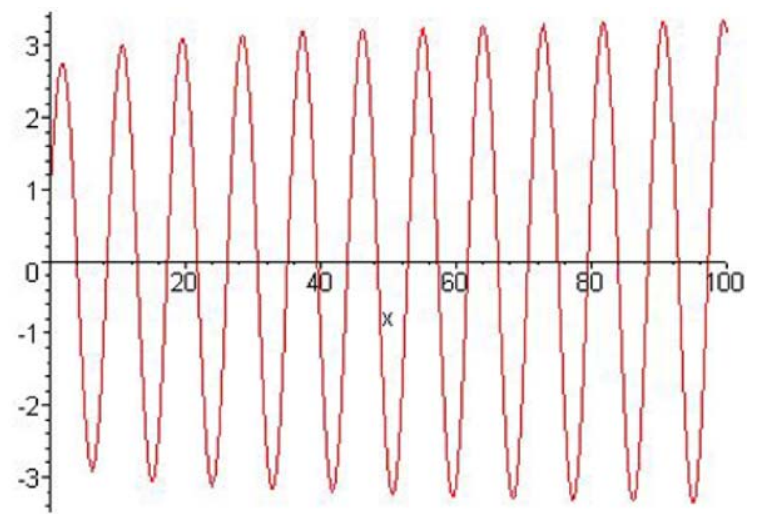

Fig. 1. Solution for $b=0, a / g=-1$ and $D=\frac{9}{10}$.

where $x \in(0 ; \infty)$, that can be rewritten as

$$
g x Z_{x x}^{\prime \prime}(x)+(D-1) Z_{x}^{\prime}(x)-a x Z(x)=0 .
$$

The corresponding solution is

$$
Z(x)=C_{1} x^{1-D / 2} J_{v}(\sqrt{-a / g} x)+C_{2} x^{1-D / 2} Y_{v}(\sqrt{-a / g} x),
$$

where $v=|1-D / 2|, J_{v}(x)$, and $Y_{v}(x)$ are the Bessel functions of the first and second kind.

The solution of Eq. (51) with the conditions

$$
Z(1)=e, \quad Z^{\prime}(1)=c
$$

has the form

$$
\begin{aligned}
Z(x)= & x^{1-D / 2} J_{D / 2-1}(\sqrt{-A} x)\left(c D \sqrt{-A} Y_{D / 2}(\sqrt{-A})-(e A) \sqrt{-A} Y_{D / 2}(\sqrt{-A})\right. \\
& \left.+c A Y_{D / 2+1}(\sqrt{-A})\right) /\left(A \sqrt { - A } \left(J_{D / 2+1}(\sqrt{-A}) Y_{D / 2}(\sqrt{-A})\right.\right. \\
& \left.\left.-Y_{D / 2+1}(\sqrt{-A}) J_{D / 2}(\sqrt{-A})\right)\right)+x^{1-D / 2} Y_{D / 2-1}(\sqrt{-A} x) \\
& \times\left(-c D J_{D / 2}(\sqrt{-A})+\sqrt{-A} c J_{D / 2+1}(\sqrt{-A})+e A J_{D / 2}(\sqrt{-A})\right) / \\
& \left(A\left(J_{D / 2+1}(\sqrt{-A}) Y_{D / 2}(\sqrt{-A})-Y_{D / 2+1}(\sqrt{-A}) J_{D / 2}(\sqrt{-A})\right)\right),
\end{aligned}
$$

where $A=a / g$.

The solutions with $b=0, g=1$, and $a / g=-1$ are demonstrated in Fig. 1 for $D=\frac{9}{10}$ and in Fig. 2 for $D=\frac{1}{10}$. The growth of solution is saturated by the nonlinear term and the full solution for (50) may appear similar to the vicinity of a stable limit cycle. 


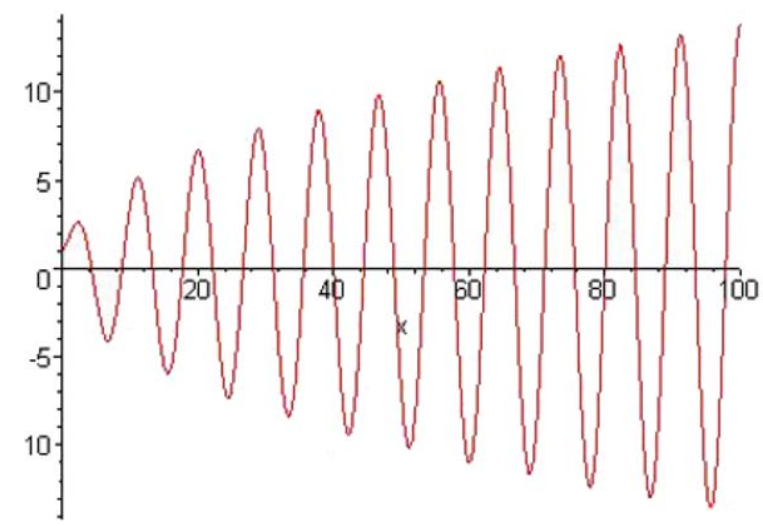

Fig. 2. Solution for $b=0, a / g=-1$ and $D=\frac{1}{10}$.

\section{Conclusion}

Derivatives and integrals of fractional order have found many applications in recent studies in physics. In this paper, we use the fractional integrals to describe fractal media with noninteger mass dimensions. Fractional integration can also be used to describe time-dependent processes in fractal media. The fractional Ginzburg-Landau equation for fractal media is derived from the corresponding generalization of free energy functional and variational Euler-Lagrange equations. Generalization of the Agrawal variational equation [14] appears to be very useful for this goal.

The fractional continuous models have wide applications. Partly, it is because a relatively small number of parameters can define a complex medium with a rich structure. In many cases, the real fractal structure of matter can be disregarded and the medium can be described by some fractional continuous mathematical model. Smoothing of the microscopic characteristics over the physically infinitesimal volume transforms the initial fractal medium into a fractional continuous model that uses the fractional integrals of the same order as the fractal mass dimension of the media. The fractional integrals can be considered as an approximation of the integrals on fractals [9]. Note that fractional integrals can be considered as integrals over the space with fractional dimension up to a numerical factor [10]. Some applications of the FGL equation were discussed also in Ref. [20].

\section{Acknowledgements}

G.M.Z. was supported by the Office of Naval Research, Grant no. N00014-02-10056, U.S. Department of Energy Grant no. DE-FG02-92ER54184, and the NSF Grant no. DMS-0417800. 


\section{References}

[1] G.M. Zaslavsky, Phys. Rep. 371 (2002) 461-580.

[2] A.I. Saichev, G.M. Zaslavsky, Chaos 7 (1997) 753-764.

[3] G.M. Zaslavsky, M. Edelman, Chaos 11 (2001) 295-305.

[4] R. Nigmatullin, Phys. Stat. Solidi B 133 (1986) 425-430.

[5] E.W. Montroll, M.S. Shlesinger, in: J. Lebowitz, E.W. Montroll (Eds.), Studies in Statistical Mechanics, vol. 11, North-Holland, Amsterdam, 1984, pp. 1-121.

[6] M.F. Shlesinger, G.M. Zaslavsky, J. Klafter, Nature 363 (1993) 31-37.

[7] G.M. Zaslavsky, Hamiltonian Chaos and Fractional Dynamics, Oxford University Press, Oxford, 2005.

[8] V.E. Tarasov, Phys. Lett. A 336 (2005) 167-174.

[9] F.Y. Ren, J.R. Liang, X.T. Wang, W.Y. Qiu, Chaos, Solitons and Fractals 16 (2003) 107-117.

[10] V.E. Tarasov, Chaos 14 (2004) 123-127.

[11] J.C. Collins, Renormalization, Cambridge University Press, Cambridge, 1984, Section 4.1

[12] H. Weitzner, G.M. Zaslavsky, Commun. Nonlinear Sci. Numer. Simul. 8 (2003) 273-281.

[13] E.M. Lifshitz, L.P. Pitaevsky, Statistical Physics, Landau Course on Theoretical Physics, vol. 9, Pergamon Press, Oxford, New York, 1980.

[14] O.P. Agrawal, J. Math. Anal. Appl. 272 (2002) 368-379.

[15] S.G. Samko, A.A. Kilbas, O.I. Marichev, Integrals and Derivatives of Fractional Order and Applications, Nauka i Tehnika, Minsk, 1987. Translation: Fractional Integrals and Derivatives Theory and Applications, Gordon and Breach, New York, 1993.

[16] V.V. Zosimov, L.M. Lyamshev, Physics-Uspekhi 38 (1995) 347-385.

[17] G.M. Zaslavsky, S.S. Abdullaev, Chaos 7 (1997) 182-186.

[18] W.A. Woyczynski, in: O.E. Berndorff-Nielsen, et al. (Eds.), Lévy Processes Theory and Applications, Birkhäuser, Boston, 2001, pp. 241-266.

[19] P. Biler, T. Funaki, W.A. Woyczynski, J. Differential Equations 148 (1998) 9-46.

[20] A.V. Milovanov, J.J. Rasmussen, Phys. Lett. A 337 (2005) 75-80. 